\title{
Rendimiento académico y deserción de estudiantes universitarios de un curso en modalidad virtual y presencial
}

\section{(Academic Achievement and Dropout of University Students from a Course in Both an Online and Face-to- Face Modality)}

\author{
Eduardo Gonzales Lopez \\ Inés Evaristo Chiyong \\ Universidad Tecnológica del Perú, UTP (Perú)
}

DOI: https://doi.org/10.5944/ried.24.2.29103

Cómo referenciar este artículo:

Gonzales Lopez, E., y Evaristo Chiyong, I. (2021). Rendimiento académico y deserción de estudiantes universitarios de un curso en modalidad virtual y presencial. RIED. Revista Iberoamericana de Educación a Distancia, 24(2), pp. 189-202. https://doi.org/10.5944/ried.24.2.29103

\section{Resumen}

La crisis sanitaria ha generado una rápida expansión de la virtualidad en la educación superior; incluso se espera que ésta mantenga una fuerte presencia, una vez superada la pandemia. Sin embargo, en Perú existe una profunda desconfianza y percepción negativa respecto de esta modalidad, por lo que se hace necesario realizar estudios que corroboren lo que en otros países ha sido encontrado: que no deberían existir diferencias en el rendimiento académico a partir de cualquier modalidad. No obstante, se ha encontrado una mayor tasa de deserción en cursos virtuales, cuando se comparan con cursos presenciales, siendo las explicaciones a este fenómeno, diversas. Teniendo todo esto en consideración, el presente trabajo busca determinar el efecto de un curso en modalidad virtual en el rendimiento académico y deserción de grupo de estudiantes universitarios de una universidad privada, en comparación con un grupo de estudiantes bajo la modalidad presencial. Para ello, se llevó a cabo un diseño cuasiexperimental con posprueba.

Los resultados respecto de cada una de las evaluaciones son variados; sin embargo, al considerar el promedio final, no se observan diferencias estadísticas significativas; de la misma manera, no se observaron diferencias respecto de la deserción, teniendo en cuenta que ésta fue pequeña en ambos grupos. Los resultados se encuentran en la misma línea de aquellos que indican que la modalidad en sí misma no es un factor determinante en ambas 
variables, sino que dependen de otros aspectos como es el caso de la labor del docente como acompañante.

Palabras clave: educación a distancia; rendimiento; deserción.

\begin{abstract}
The health crisis has generated a rapid expansion of virtuality in higher education; it is even expected that it will maintain a strong presence once the crisis is finished. However, in Peru there is a deep mistrust and negative perception regarding this modality. For this reason, it is necessary to carry out studies that corroborate what has been found in other countries: that there should be no differences in academic performance based on any modality. Nevertheless, a higher dropout rate has been found in virtual courses, when compared with face-to-face courses, and the explanations for this phenomenon are diverse. Taking all this into consideration, this current research aims to determine the effect of a course in virtual modality on the academic performance and dropout of a group of university students from a private university, compared to a group of students in the face-to-face modality. To do this, a quasi-experimental design with post-test was carried out.

The results regarding each of the evaluations are varied; however, when considering the final average, no statistically significant differences are observed; in the same way, no differences were observed related to dropout, regarding that this difference was small in both groups. The outcomes are in the same line as those that indicate that the modality itself is not a determining factor in both variables, but rather depend on other aspects such as the teachers' work as a guide.
\end{abstract}

Keywords: online education; achievement; dropout.

Es innegable que en los últimos años la educación virtual se ha expandido ampliamente, evidenciando un potencial importante para ayudar a superar aquellos retos que la educación superior latinoamericana supone, brindando una ampliación del acceso a la educación, mejoras en la calidad de la misma, así como la conexión entre el sistema educativo y las demandas del mercado de trabajo (OECD, 2017); además, a través de la educación virtual, existe mayor flexibilidad en cuanto a tiempo y espacio para los estudiantes, facilita el acceso a la información, es más rentable y puede llegar a ser más personalizado que otras modalidades (Arkorful y Abaidoo, 2015).

A partir del impacto de la pandemia por el COVID-19, la educación a distancia se ha presentado como la única solución para garantizar la continuidad del servicio educativo, aunque es evidente que no todos los países y, en específico, las instituciones, tuvieron los recursos para poder implementarla asegurando un nivel de calidad suficiente, de ahí que es usual referirse a esta modalidad como educación remota de emergencia (Pedró, 2020). A pesar de ello, las instituciones educativas 
han empezado a intensificar sus esfuerzos para que la modalidad a distancia deje de ser solo "de emergencia" y permita que los estudiantes participen de una óptima experiencia de aprendizaje, equiparable a la educación presencial. Así pues, incluso superada esta situación de pandemia es muy probable que la implementación de programas en modalidad virtual sea una prioridad para las instituciones educativas.

En el Perú la situación no es muy alentadora en comparación de muchos países latinoamericanos, los cuales, en general, contaban con mayor cantidad de programas semipresenciales o a distancia, antes de la pandemia (Barrios Ipenza y Olivo Chang, 2019). Además, aún existe una amplia desconfianza respecto de este tipo de educación, así como la percepción de tener una calidad inferior respecto de la educación presencial; incluso, muchos padres y estudiantes sostienen que este tipo de educación implica costos inferiores y, por tanto, se requieren mayores reducciones en las tasas de matrículas, por cuenta del Gobierno o de las mismas universidades. De esta manera, padres de familia y estudiantes han presentado numerosos reclamos a los centros de estudio y la Superintendencia Nacional de Educación Superior Universitaria (SUNEDU). A finales de abril del año 2020, esta entidad informó haber recibido 252 denuncias y más de 3000 consultas. Un tercio de ellas, aproximadamente, están relacionadas al dictado de clases en modalidad no presencial (Figallo et al., 2020).

En general, el aprendizaje virtual se puede definir como el acceso a experiencias de aprendizaje a través del uso de las tecnologías (Benson, 2002; Carliner, 2004; Conrad, 2002, como se citaron en Moore et al., 2010) y puede incorporar tanto trabajo individual y colaborativo, así como aprendizaje sincrónico y asincrónico (Zeitoun, 2008, como se citó en Arkorful y Abaidoo, 2015). Si bien una de las principales diferencias de la modalidad virtual respecto de la presencial es el espacio en donde se imparte la instrucción, no se debe soslayar la importancia de las estrategias utilizadas por el docente, quien debe realizar un conjunto de acciones específicas para asegurar el éxito de su curso; así pues, no basta con el mero cambio de modalidad. Por ejemplo, dentro de las estrategias de enseñanzaaprendizaje más efectivas para la formación virtual, algunos autores destacan el uso de la retroalimentación oportuna y de calidad, tanto individual como colectiva (Martínez-Argüelles et al., 2015), mantener una secuencia clara de las actividades a realizar, monitorear la entrega de tareas y hacer seguimiento a los estudiantes sobre ello (Ragan, 2012), fomentar la participación multidireccional, e incluir espacios sincrónicos, como las videoconferencias (Bautista Pérez et al., 2006). En el presente estudio, se ha procurado incorporar estas buenas prácticas.

Si bien la educación virtual ofrece posibilidades prometedoras, es necesario conocer si los resultados de ésta son equiparables a los resultados obtenidos en la educación presencial; es decir, necesitamos responder a la pregunta ¿qué modalidad tiene los mejores efectos en el rendimiento académico de los estudiantes?

En el Perú, hay escasa información al respecto: en un reciente estudio, no se encontraron diferencias significativas entre los resultados de un curso presencial 
y otro semipresencial (Dasso Vassallo y Evaristo Chiyong, 2020); sin embargo, no se han reportado estudios en donde se comparen cursos presenciales con cursos totalmente virtuales. En otros países, se han realizado numerosas investigaciones comparando ambas modalidades en las últimas décadas. Los resultados varían de acuerdo con el tipo de curso, las características de los estudiantes y la instrucción que se brindó (Slater, 2004); pero, en general, los hallazgos indican que no existen diferencias significativas al utilizar diferentes modalidades.

Bailey (2020) comparó las modalidades presencial, híbrido y virtual en una universidad pública norteamericana respecto de tres variables: el logro académico, la persistencia y la satisfacción de los estudiantes, los resultados apoyan la literatura existente que refiere que no existen diferencias significativas, sugiriendo que los administradores e instructores deben continuar ampliando el acceso a cursos de modalidad virtual y semipresencial.

En el 2019, Ebner y Gegenfurtner encontraron que los webinars (espacios sincrónicos) son, a nivel descriptivo, más efectivos que el aprendizaje presencial y que la instrucción asincrónica online; sin embargo, las diferencias entre los webinars y las otras dos modalidades fueron marginales y estadísticamente no significativas. En resumen, se podría asumir que las tres modalidades tienden a ser igualmente efectivas para el aprendizaje.

Por su parte, Soffer y Nachmias (2018) en una universidad de Tel Aviv, compararon la efectividad de 3 cursos en línea con los mismos cursos en formato presencial con similares características. Los resultados indican que existen diferencias significativas entre ambos. Los estudiantes de la modalidad virtual reportaron mejor comprensión de la estructura del curso, mejor comunicación con el personal del curso, mayor compromiso y satisfacción. Sin embargo, los estudiantes de la modalidad presencial reportaron que hubo una mejor contribución del curso a su aprendizaje. Finalmente, las calificaciones de los estudiantes de la modalidad virtual fueron más altas, aunque no hubo diferencias en la tasa de completamiento. Estos hallazgos contribuyen a la noción que, en muchos de los aspectos examinados, los cursos virtuales son tan o más efectivos que los presenciales.

Cooper et al. (2006, como se citó en Clark y Mayer, 2016) también coinciden en que la educación virtual y presencial pueden ser igual de efectivas y, más bien, una de las variables que tiene mayor impacto en el aprendizaje, es la calidad de la instrucción. De esta manera, es más probable que un estudiante que participa de un curso en línea bien diseñado aprenda de manera significativa y más efectiva en comparación con un curso pobremente planificado e implementado. En general, la evidencia parece indicar que son los métodos instruccionales aquellos que tienen mayor efecto en el aprendizaje y no la modalidad (Clark, 2001, como se citó en Clark y Mayer, 2016).

Además del rendimiento académico, la deserción es otra variable importante para analizar cuando se compara la educación virtual con la presencial; esta es un fenómeno con consecuencias negativas que afecta diferentes niveles de la persona 
y su entorno. Por ejemplo, aquellos estudiantes que no concluyen sus estudios son subempleados y no pueden obtener los ingresos económicos que esperan (González y Girón, 2005). En el caso del Perú, los niveles de deserción universitaria tienen una alta heterogeneidad, con instituciones de gran población universitaria presentando altas tasas de deserción, independientemente de la modalidad (Barrios Ipenza y Olivo Chang, 2019).

La deserción es un fenómeno complejo multideterminado, Vázquez y Rodríguez (2007, como se citó en La Madriz, 2016), consideran los siguientes factores relacionados con la deserción: la integración social y compromiso institucional e individual del estudiante, su capacidad intelectual, compromiso académico e identificación profesional, así como los factores socioeconómicos, educativos y demográficos. En esta misma línea, se ha reportado que los estudiantes peruanos que interrumpieron sus estudios universitarios, pero los retomaron, tuvieron como principales razones las dificultades económicas, trabajo, motivos familiares y enfermedad (Barrios Ipenza y Olivo Chang, 2019).

Algunos autores como Álvarez y López (2017) y Vásquez y Rodríguez (2007) y Cho y Heron, 2015; Frankola 2001; Hachey et al. (2013, como se citaron en Grau-Valldosera et al., 2019) han comparado la deserción en la modalidad virtual y presencial, concluyendo que ésta es mayor en la modalidad virtual que en la presencial. Si bien no se tienen datos en el contexto peruano, en otros países latinoamericanos como Colombia, la tasa de abandono en programas presenciales es del 48\%, mientras que en programas a distancia es del 60\% (MEN, 2009). Esto podría explicarse debido a las diferencias entre la metodología de ambas modalidades, así como los diferentes perfiles; específicamente, los estudiantes de la modalidad virtual suelen ser adultos que trabajan con obligaciones familiares $y$, por ende, cuentan con menor tiempo para los estudios. Escanés et al. (2014) indican que muchos estudiantes que escogen esta modalidad pueden presentar dificultades de gestión del tiempo, las cuales podrían estar influenciadas por sus obligaciones familiares y/o laborales producto de dificultades económicas y/o un ingreso tardío a la educación universitaria. Si estas problemáticas de índole personal se mantienen, si se agravan las limitaciones que estas dificultades implican para el desarrollo de sus proyectos educativos personales, o si se debilitan mecanismos que les permitan sobrellevarlas, la deserción está prácticamente asegurada.

Sintetizando las posibles causas de la deserción en modalidad virtual, Lee y Choi (2011) identificaron tres macro factores, los cuales se mencionan en orden de frecuencia. En primer lugar, se encuentran los factores del estudiante, dentro de los cuales están los atributos psicológicos, habilidades y experiencias relevantes, así como antecedentes académicos. En segundo lugar, se encuentran los factores ambientales, dentro de los que se encuentran los ambientes de apoyo para el estudio y compromisos laborales. Finalmente, se encuentran los factores relacionados con el curso, dentro de los que se hallan las interacciones, apoyo institucional y diseño del curso. 
En conclusión, si bien la investigación internacional indica que las diferencias respecto del rendimiento académico entre ambas modalidades se pueden deber a variables como la calidad de las estrategias y no al propio medio y que la educación virtual parece tener mayores tasas de deserción, en el Perú no existen investigaciones que puedan corroborarlo. A esto se suma la percepción de que la educación en línea es, en general, inferior a la modalidad presencial. Considerando lo ya mencionado, la presente investigación busca responder a la pregunta, ¿Cuál es el efecto de un curso en modalidad virtual en el rendimiento académico y deserción de grupo de estudiantes universitarios de una universidad privada, en comparación con un grupo de estudiantes bajo la modalidad presencial?

\section{METODOLOGÍA}

\section{Diseño}

El alcance de este trabajo fue explicativo, con un diseño cuasiexperimental debido a que se manipuló una variable independiente para observar su efecto sobre dos variables dependientes con grupos intactos (Hernández et al., 2014); específicamente, se utilizó un diseño con posprueba únicamente y grupo de control; de esta manera, al concluirse la manipulación de la variable independiente, a ambos grupos se les administró una medición sobre las variables dependientes en estudio.

Para esta investigación, se consideró como variable independiente el curso Estadística Descriptiva y Probabilidades bajo la modalidad virtual. Las variables dependientes fueron el rendimiento académico y la deserción del curso; el rendimiento se operacionalizó como las calificaciones obtenidas por los estudiantes en todas las actividades de evaluación sumativa del curso; es decir, tres prácticas, una evaluación parcial, un examen final, un trabajo final y la evaluación permanente, así como el promedio final. Por su parte, la deserción del curso se operacionalizó como la cantidad de alumnos que dejaron de asistir a las clases y no se reincorporaron durante el semestre, así como aquellos estudiantes que formalizaron su retiro.

\section{Participantes}

La selección de los participantes fue conformada a partir de un muestreo intencional, quienes fueron estudiantes del primer ciclo del curso Estadística Descriptiva y Probabilidades de una universidad privada con sede en Lima. Es importante mencionar que los estudiantes pertenecientes al grupo experimental no tuvieron experiencia previa en la modalidad virtual, por lo menos en la institución en donde se realizó el estudio.

Para el análisis de la deserción, se trabajó con un total de 39 estudiantes asignados para el grupo control, de los cuales fueron 35 hombres y 4 mujeres, mientras que en el 
grupo experimental se contó con 28 estudiantes, de los cuales fueron 2 mujeres y 26 hombres. Respecto del análisis del rendimiento académico, no se consideraron a los estudiantes retirados, por lo que se contó con un total de 37 estudiantes asignados al grupo control, de los cuales fueron 4 mujeres y 33 hombres; mientras que en el grupo experimental fueron 24 estudiantes, de los cuales fueron 2 mujeres y 22 hombres.

\section{Instrumentos}

La variable de rendimiento académico se midió utilizando las calificaciones asignadas a cada estudiante de acuerdo con el registro de notas. Para medirla se utilizó la calificación vigesimal, haciendo un total de 20 puntos.

Las evaluaciones consistieron en lo siguiente: las prácticas, el examen parcial y final implicaron el análisis de casos realizados de manera individual, con respuestas correctas únicas y llevadas a cabo de manera sincrónica. Por su parte, el trabajo final fue una actividad colaborativa, realizada a lo largo de varias semanas de manera asincrónica, para luego ser presentada de manera sincrónica al final del semestre. Finalmente, la evaluación permanente consistió en la calificación asignada a las participaciones de los estudiantes, revisión de los contenidos virtuales y obtención de las insignias digitales. Por su parte, la deserción fue medida a través del registro de asistencia, así como los reportes formales de retiro del curso.

\section{Procedimiento}

Para crear el curso virtual, se realizó una adaptación del curso en modalidad presencial, manteniendo los objetivos y contenidos, ajustándose tanto las estrategias instruccionales como los recursos de aprendizaje. Así pues, se diseñaron videos sobre el contenido temático semanal con el mismo docente dictante y se adaptaron también las evaluaciones presenciales a la modalidad virtual. Adicionalmente, se incorporó un programa de insignias digitales, el cual consistió en asignar monedas y medallas virtuales a los estudiantes que cumplían con una serie de actividades. Todo ello se implementó en la plataforma virtual educativa Canvas.

Respecto de las funciones del docente virtual, se le asignó un conjunto de acciones semanales a realizar; incluyendo el envío de anuncios, moderación del foro de consultas, revisión de las actividades y realización de videoconferencias. Para ello, fue previamente capacitado.

Es importante mencionar que ambas versiones del curso tuvieron una duración de 14 semanas y el mismo docente estuvo a cargo de ambas. Además, esta investigación se realizó antes del contexto de la pandemia producida por el COVID-19. 


\section{RESULTADOS}

Para realizar el análisis estadístico de ambos grupos se utilizó el paquete estadístico SPSS (Statistical Package for Social Sciences) versión 25. Primero se realizó el análisis del rendimiento académico, cuyos resultados descriptivos se pueden observar en la tabla 1. Respecto de los análisis inferenciales, se corroboró el cumplimiento de las condiciones necesarias para poder llevar a cabo la comparación de muestras independientes usando una prueba paramétrica (Coolican, 2014). Para comprobar el supuesto de normalidad, se utilizó el análisis de Shapiro-Wilks; sin embargo, los resultados indican que los puntajes de ambos grupos no se aproximan a una distribución normal debido a que los valores de p fueron, en su mayoría, inferiores a 0.001. Considerando estos resultados, se decidió utilizar la prueba no paramétrica U de Mann-Whitney.

En el caso de la práctica 1, se rechaza la hipótesis nula, por lo que se concluye que la distribución de las medias es diferente en ambos grupos, a favor del grupo experimental; ello debido a que p<0.0001. Adicionalmente, se halló el tamaño del efecto para cada una de las evaluaciones, éste se refiere al tamaño de la diferencia o relación que se encuentra en el estudio (Howitt y Cramer, 2017). Así pues, para la práctica 1, el tamaño del efecto fue grande, debido a que $\mathrm{g}=0.99$. En el caso de la práctica 2, se acepta la hipótesis nula debido a que la distribución de las medias es igual entre ambos grupos, teniendo en cuenta que $\mathrm{p}=0.082$, mientras que el tamaño del efecto fue pequeño, debido a que $\mathrm{g}=0.25$.

Respecto de la evaluación parcial, se rechaza la hipótesis nula que indica que la distribución de las medias es la misma en el grupo control y experimental, debido a que $\mathrm{p}=0.040$. Así pues, se encontró una diferencia a favor del grupo experimental. Por su parte, el tamaño del efecto fue pequeño, debido a que $\mathrm{g}=\mathbf{0 . 2 1}$. Teniendo en cuenta la práctica 3 , se acepta la hipótesis nula que indica que la distribución es la misma entre ambos grupos, debido a que $\mathrm{p}=0.309$. Por su parte, el tamaño del efecto es pequeño debido a que $\mathrm{g}=0.20$.

Considerando el trabajo final, se rechaza la hipótesis nula que indica que la distribución de las medias es igual, debido a que p<0.0001; así pues, la diferencia encontrada fue a favor del grupo control, mientras que el tamaño del efecto fue grande debido a que $g=1.27$. Respecto de la evaluación permanente, se rechaza la hipótesis nula que indica que la distribución de las medias es la misma, debido a que $\mathrm{p}<0.001$, con una diferencia a favor del grupo control. Además, el tamaño del efecto fue grande, debido a que $\mathrm{g}=0.85$.

En el caso del examen final, los resultados nos llevan a aceptar la hipótesis nula, la cual indica que la distribución de las medias es la misma para ambos grupos, debido a que $\mathrm{p}=0.38$. Además, el tamaño del efecto fue de $\mathrm{g}=0.15$. Por último, respecto del promedio final, los resultados nos llevan a aceptar la hipótesis nula, la cual indica que la distribución de las medias en ambos grupos es igual, pues la probabilidad es 
0.34, superior al .05. Además, el tamaño del efecto fue pequeño, debido a que g= 0.32 .

\section{Tabla 1}

Estadísticos descriptivos respecto del promedio final

\begin{tabular}{|c|c|c|c|c|c|c|c|c|c|}
\hline \multicolumn{2}{|c|}{ Grupo } & $\begin{array}{l}\text { Prome- } \\
\text { dio final }\end{array}$ & $\begin{array}{c}\text { Examen } \\
\text { final }\end{array}$ & $\begin{array}{c}\text { Evaluac. } \\
\text { perm }\end{array}$ & $\begin{array}{c}\text { Trabajo } \\
\text { final }\end{array}$ & $\begin{array}{c}\text { Prác. } \\
\mathbf{3}\end{array}$ & Parc & $\begin{array}{c}\text { Prác. } \\
2 \\
\end{array}$ & $\begin{array}{c}\text { Prác. } \\
1\end{array}$ \\
\hline \multirow{6}{*}{ Control } & Media & 13.51 & 8.33 & 16.14 & 16.03 & 11.97 & 14.14 & 15.16 & 14.27 \\
\hline & Mediana & 14.00 & 8.00 & 18.00 & 18.00 & 14.00 & 15.00 & 16.00 & 15.00 \\
\hline & Varianza & 7.04 & 20.10 & 24.38 & 18.81 & 28.80 & 11.07 & 12.64 & 12.04 \\
\hline & Desv. & 2.65 & 4.48 & 4.94 & 4.34 & $5 \cdot 37$ & 3.33 & 3.56 & 3.47 \\
\hline & Mínimo & 3.00 & 0.01 & 0.01 & 0.00 & 0.01 & 7.00 & 6.00 & 4.00 \\
\hline & Máximo & 16.00 & 19.00 & 20.00 & 19.00 & 19.00 & 20.00 & 20.00 & 20.00 \\
\hline \multirow{6}{*}{$\begin{array}{l}\text { Experi- } \\
\text { mental }\end{array}$} & Media & 12.38 & 9.21 & 11.88 & 8.96 & 13.13 & 15.13 & 16.21 & 17.67 \\
\hline & Mediana & 13.50 & 12.00 & 12.50 & 7.00 & 15.00 & 18.00 & 18.00 & 20.00 \\
\hline & Varianza & 22.33 & 53.51 & 25.51 & 49.69 & 39.92 & 40.28 & 25.12 & 11.01 \\
\hline & Desv. & 4.73 & $7 \cdot 31$ & 5.05 & 7.05 & 6.32 & 6.35 & 5.01 & $3 \cdot 32$ \\
\hline & Mínimo & 1.00 & 0.01 & 3.00 & 0.01 & 0.01 & 0.00 & 0.01 & 8.00 \\
\hline & Máximo & 19.00 & 19.00 & 19.00 & 18.00 & 20.00 & 20.00 & 20.00 & 20.00 \\
\hline
\end{tabular}

Fuente: elaboración propia

Respecto de la deserción, fueron solo 2 los estudiantes retirados del grupo control y 4 del experimental; así pues, las cantidades fueron bastante bajas en ambas modalidades. Aun así, se realizó el análisis inferencial utilizando la prueba exacta de Fisher, debido a que ambas variables eran nominales y la frecuencia esperada en dos de las cuatro celdas es menor de 5 . Con los resultados obtenidos se comprueba la hipótesis nula de que las dos variables son independientes, debido a que: el valor de $\mathrm{p}=0.390$ es menor que el nivel de significación 0.05 .

\section{Tabla 2}

Comparación entre modalidades respecto de alumnos matriculados y retirados

\begin{tabular}{lcc} 
& Control & Experimental \\
\hline Estudiantes matriculados & 39 & 28 \\
\hline Estudiantes retirados & 2 & 4 \\
\hline Cantidad de estudiantes que finalizaron el curso & 37 & 24 \\
\hline
\end{tabular}

Fuente: elaboración propia 


\section{DISCUSIÓN}

En general, se pueden observar diferencias respecto del rendimiento de los estudiantes en algunas de las evaluaciones realizadas; sin embargo, esta diferencia no se refleja al considerar el promedio final, lo cual permite inferir que los aprendizajes fueron similares. En ambos grupos se puede observar que las calificaciones son heterogéneas: hay una varianza elevada en ambos grupos, pero mayor en el experimental. Así pues, hubo estudiantes que alcanzaron puntuaciones promedio tan bajas como 1. Al indagar con el docente por estos casos, refirió que son alumnos cuya situación era incierta, debido a que mantuvieron presencia inconstante en el curso y la comunicación con ellos fue difícil de establecer. Se sospecha que estos alumnos pudieron haber presentado situaciones personales y/o familiares no informadas al docente, que les impidieron rendir apropiadamente sus evaluaciones y, progresivamente, su participación en el curso fue decayendo; así también, se infiere que tuvieron dificultades serias de conectividad. Sobre este último punto, vale la pena mencionar que, al inicio del curso se identificó que el 70\% de los alumnos encuestados contaban con equipos que cumplían con procesadores y memoria mínimos para poder participar de las videoconferencias, siendo este un indicador de su bajo nivel económico. Esto tiene relación con lo mencionado por Ferreyra et al. (2017), quienes indican que las características promedio de los individuos que ingresan a la educación superior en los últimos años han cambiado: hay mayor presencia de estudiantes con menores ingresos económicos, con mayores probabilidades de tener una preparación académica menor y ser los primeros en su familia en acceder a la educación superior.

Centrándonos en cada una de las actividades de evaluación, en la primera práctica calificada se observa una diferencia significativa y tamaño del efecto grande a favor del grupo experimental, esto podría explicarse debido al efecto de la novedad del formato virtual, siendo esta la primera actividad calificada, lo cual podría corroborarse por la disminución del promedio de notas obtenido por el grupo experimental hasta la quinta evaluación.

Otra diferencia significativa con tamaño del efecto grande se dio en el trabajo final, en donde el grupo control obtuvo mejores calificaciones. Una posible explicación a estos resultados tiene que ver con el menor tiempo que el docente dedicó a las asesorías sincrónicas previas a la actividad final en la modalidad virtual; así pues, esto ocurrió debido a que la carga de trabajo fue mayor en el curso virtual, específicamente, el docente indicó que el aprendizaje de estrategias instruccionales virtuales, el uso de la plataforma educativa y otras herramientas virtuales, así como el diseño y desarrollo de su aula virtual, le significó un incremento importante en el tiempo dedicado al curso. Considerando que el trabajo final requería de retroalimentación continua, esta reducción del tiempo dedicado al asesoramiento pudo ser una variable importante que afectó el rendimiento de los estudiantes. Esto concuerda con lo referido por Ward et al. (2010), quienes indicaron que los 
estudiantes universitarios de su estudio percibieron las clases con comunicación sincrónica con una mayor calidad instruccional en comparación con otros métodos asincrónicos.

Los resultados del grupo experimental también pudieron haberse influenciado por otras variables no controladas, las cuales pudieron afectar el rendimiento académico de este grupo; por ejemplo, debido a que las evaluaciones se realizaron de manera virtual, no se pudo eliminar en su totalidad la probabilidad de plagio; si bien se tomaron algunas medidas para evitar ello, como la aleatorización de las preguntas y el uso del tiempo límite, éstas pueden resultar insuficientes, teniendo en cuenta que, de manera global, el uso del internet parece incrementar el plagio (Lyon et al., 2006, como se citó en Youmans, 2011).

A pesar de las limitaciones del presente trabajo, estos resultados apoyan lo afirmado por Dell et al. (2010), quienes refieren que hay una tendencia en los estudios de este tipo que lleva a concluir que el rendimiento de los estudiantes se ve influenciado más por el diseño y las estrategias utilizadas que de la modalidad. Con diseños rigurosos, independientemente de la modalidad, los resultados no deberían diferir significativamente.

Respecto de la deserción, la cantidad de estudiantes retirados en ambos cursos fue pequeña, pero ligeramente mayor en el grupo experimental; sin embargo, los resultados del presente trabajo son insuficientes para poder corroborar que, efectivamente, la deserción es significativamente mayor en la modalidad virtual en comparación con la presencial.

Analizando con mayor detalle estos casos específicos, se encontró que estos alumnos fueron parte del grupo de estudiantes que no contaban con los requisitos mínimos para llevar a cabo las sesiones sincrónicas de manera apropiada, incluso, algunos de ellos se encontraban dentro del 12,9\% de estudiantes que disponía del smartphone como único medio para participar de sus clases virtuales. Debemos tener en cuenta que los participantes de esta investigación no estaban llevando su carrera en modalidad virtual ni semipresencial, sino más bien presencial, siendo esta una experiencia piloto, por lo que es comprensible que muchos estudiantes no cuenten con los recursos mínimos para esta modalidad. Esto refleja la importancia de comunicar a los estudiantes que se matriculan, qué recursos y conocimientos se espera que tengan para participar de manera óptima del proceso de aprendizaje en modalidad virtual.

Asimismo, es probable que también hayan influenciado otras variables de tipo económico, laboral social, familiar, emocional, etc. Por ejemplo, se identificó que por lo menos el $49 \%$ de los estudiantes se encontraban trabajando a tiempo completo, contando con menos tiempo durante la semana para dedicarse exclusivamente a sus cursos.

Si bien Lee y Choi (2011) consignaron los factores del estudiante como las más frecuentes al momento de explicar la deserción, en este estudio se han identificado más bien las variables ambientales como las que podrían tener un mayor impacto. 
Asimismo, considerando que esta propuesta es de hace casi una década, es natural que las variables personales relacionadas con las habilidades tecnológicas no tengan tanto impacto ahora, considerando que los estudiantes hacen uso frecuente de herramientas tecnológicas.

En conclusión, este trabajo se encuentra en la misma línea de otros estudios, en tanto la modalidad no parece ser un factor determinante al momento de asegurar el rendimiento académico de los estudiantes, sino más bien la labor del instructor, en especial como tutor acompañante, así como de las estrategias que usa. En el caso de la deserción, si bien no hubo diferencias significativas, el análisis pareciera indicar que las variables no pedagógicas juegan un rol muy importante en la permanencia de los alumnos, por lo que queda para futuras investigaciones explorar con mayor profundidad las diferencias entre ambas modalidades y las variables que pueden estar causando un mayor impacto.

\section{REFERENCIAS}

Álvarez, P. R., y López, D. (2017). Estudios sobre deserción académica y medidas orientador de prevención en la Universidad de La Laguna (España). Revista Paradigma, 38(1), 48-71.

Arkorful, V., y Abaidoo, N. (2015). The role of e-learning, advantages and disadvantages of its adoption in higher education. International Journal of Instructional Technology \& Distance Learning, 12(1), 29-43.

Bailey, L. M. (2020). Comparing Students' Learning Outcomes and Satisfaction in Online, Hybrid and Face-To-Face Education Courses. Dissertation, Temple University. https://scholarshare.temple.edu/ bitstream/handle/20.500.12613/292/ Bailey temple 0225E 14160 . pdf?sequence $=1$ \&isAllowed $=\mathrm{y}$

Barrios Ipenza, E., y Olivo Chang, D. (2019). La educación a distancia como oportunidad de equidad y democratización en el Perú. En C. Rama Vitale, y M. Morocho Quezada (Edits.), Prospectiva de la Educación a Distancia en América Latina y el Caribe (pp. 167-168). EDILOJA Cía. Ltda. http:// www.caled-ead.org/sites/default/files/
files/Prospectiva-Educacio\%CC\%81n. pdf\#page $=167$

Bautista Pérez, G., Borges Sáiz, F., y Forés, A. (2006). Didáctica universitaria en Entornos Virtuales. Narcea.

Clark, R. C., y Mayer, R. E. (2016).e-Learning and the Science of Instruction. Proven Guidelines for Consumers and Designers of Multimedia Learning (Fourth ed.). John Wiley \& Sons, Inc. https://doi. org/10.1002/9781119239086

Coolican, H. (2014). Research Methods and Statistics in Psychology (Sixth ed.). Routledge. https://doi. org/10.4324/9780203769669

Dasso Vasallo, A., y Evaristo Chiyong, I. (2020). Análisis de resultados del aprendizaje presencial y aprendizaje semipresencial en dos cursos universitarios. Educación, 29(57), 27-42. https://doi.org/10.18800/ educacion.202002.002

Dell, C. A., Low, C., y Wilker, J. F. (2010). Comparing Student Achievement in Online and Face-to-Face Class Formats. MERLOT Journal of Online Learning and Teaching, 6(1), 30-42.

Ebner, C., y Gegenfurtner, A. (2019). Learning and Satisfaction in Webinar, 
Online, and Face-to-Face Instruction: A Meta-Analysis. Front. Educ., 4(92), 1-11. https://doi.org/10.3389/ feduc.2019.00092

Escanés, G., Herrero, V., Merlino, A., y Ayllón, S. (2014). Deserción en educación a distancia: factores asociados a la elección de modalidad como desencadenantes del abandono universitario. Virtualidad, Educación y Ciencia, 5(9), 45-55.

Ferreyra, M., Avitabile, C., Botero, J., Haimovich, F., y Urzúa, S. (2017). At a Crossroads: Higher Education in Latin America and the Caribbean. Directions in Development. World Bank. https://doi. org/10.1596/978-1-4648-0971-2

Figallo, F., González, M. T., y Diestra, V. (2020). Perú: Educación Superior en el Contexto de la Pandemia por el COVID-19. ESAL - Revista de Educación Superior en América Latina, 20-28. https://doi. org/10.14482/esal.8.378.85

González, D. y Girón, L. (2005). Determinantes del rendimiento académico y la deserción estudiantil en el programa de Economía de la Pontificia Universidad Javeriana de Cali. Economía, Gestión y Desarrollo, 3, 173-201.

Grau-Valldosera, J., Miguillón, J., y BlascoMoreno, A. (2019). Returning after taking a break in online distance higher education: from intention to effective re-enrolment. Interactive Learning Environments, 27(3), 307-323. https:// doi.org/10.1080/10494820.2018.147098 $\underline{6}$

Hernández, R., Fernández, C., y Baptista, P. (2014). Metodología de la investigación (Sexta ed.). McGraw-Hill / Interamericana Editores, S.A. de C.V.

Howitt, D., y Cramer, D. (2017). Research Methods in Psychology (Fifth ed.). Pearson Education Limited.

La Madriz, J. (2016). Factores que promueven la deserción del aula virtual. Revista Científica Electrónica de Ciencias Humanas, 35(12), 18-40.
Lee, Y., y Choi, J. (2011). A review of online course dropout research: implications for practice and future research. Educational Technology Research and Development, 59, 593-618. https://doi.org/10.1007/ s11423-010-9177-y

Martínez-Argüelles, M. J., Plana, D., Hintzmann, C., Batalla-Busquets, J.M., y Badia, M. (2015). Usefulness of feedback in e-learning from the students' perspective. Intangible Capital, 11(4), 627-645. https://doi.org/10.3926/ic.622

Ministerio de Educación Nacional (2009). Deserción Estudiantil en la Educación superior colombiana. Metodología de seguimiento, diagnóstico y elementos para su prevención. Revolución Educativa. MEN.

Moore, J. L., Dickson-Deane, C., y Galyen, K. (2010). e-Learning, online learning, and distance learning environments: Are they the same? Internet and Higher Education. https://doi.org/10.1016/j. iheduc.2010.10.001

OECD. (2017). La educación a distancia en la educación superior en América Latina.

Pedró, F. (2020). Covid-19 y educación superior en América Latina y Caribe: Efectos, impactos y recomendaciones políticas. Fundación Carolina, 1-15. https://doi.org/10.33960/AC 36.2020

Ragan, L. (2012). 10 Principles of Effective Online Teaching: Best Practices in Distance Education. Faculty Focus. Magna.

Slater, B. (2004). A comparison of online and face-to-face instruction in an undergraduate. Contemporary Issues in Technology and Teacher, 4(2), 196-213.

Soffer, T., y Nachmias, R. (2018). Effectiveness of learning in online academic courses compared with faceto-face courses in higher education. Journal of Computer Assisted Learning, 34(5), 535-543. https://doi.org/10.1111/ jcal.12258 
Vásquez, C. R., y Rodríguez, M. C. (2007). La deserción estudiantil en educación superior a distancia: perspectiva teórica y factores de incidencia. Revista Latinoamericana de Estudios Educativos, 37(3-4), 107-122.

Ward, M. E., Peters, G., y Shelley, K. (2010). Student and Faculty Perceptions of the Quality of Online Learning Experiences.
International Review of Research in Open and Distance Learning, 11(3), 57-77. https://doi.org/10.19173/irrodl.v11i3.867

Youmans, R. J. (2011). Does the adoption of plagiarism-detection software in higher education reduce plagiarism? Studies in Higher Education, 1-13. https://doi.org/ $\underline{10.1080 / 03075079.2010 .523457}$

\section{PERFIL ACADÉMICO Y PROFESIONAL DE LOS AUTORES}

Eduardo Gonzales Lopez. Licenciado en Psicología por la Universidad Nacional Mayor de San Marcos y Magíster en Docencia Universitaria y Gestión Educativa por la Universidad Tecnológica del Perú. Especialista en Diseño Curricular de la Facultad de Gestión de la Pontificia Universidad Católica del Perú. Sus líneas de investigación se centran en programas formativos semipresenciales y virtuales en educación superior. En su trabajo reciente ha profundizado en el uso de las insignias digitales. https://orcid.org/0000-0002-4097-0613

E-mail: egonzaleslopez@gmail.com

Inés Evaristo Chiyong. Licenciada en Psicología Educacional por la Pontificia Universidad Católica del Perú (PUCP). Magíster en Enseñanza y Aprendizaje Abiertos y a Distancia por la UNED-España. Egresada de la Maestría en Políticas Educativas por la PUCP. Directora de Aprendizaje Virtual de la Universidad Tecnológica del Perú. Docente del Departamento de Psicología PUCP. Sus líneas de investigación se centran en el aprendizaje virtual, educación a distancia, innovación educativa y metodologías de aprendizaje. https://orcid.org/0000-0003-2308-4289

E-mail: ievaristo@inlearning.edu.pe

DIRECCIÓN DE LOS AUTORES

Universidad Tecnológica del Perú

Jirón Natalio Sánchez 125

Lima (Perú)

Fecha de recepción del artículo: 15/12/2020

Fecha de aceptación del artículo: 14/03/2021

Fecha de aprobación para maquetación: 18/03/2021 